\title{
Immediate carotid endarterectomy reduced non-perioperative stroke in severe asymptomatic carotid artery stenosis
}

Halliday A, Mansfield A, Marro J, et al. Prevention of disabling and fatal strokes by successful carotid endarterectomy in patients without recent neurological symptoms: randomised controlled trial. Lancet 2004;363:1491-502.

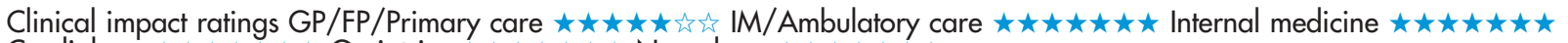

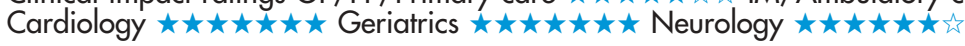

\section{In patients with severe carotid artery stenosis but no recent $(\leqslant 6 \mathrm{mo})$ stroke or ischaemia, is immediate carotid endarterectomy (CEA) more effective than indefinite deferral of any CEA for reducing perioperative mortality and morbidity and incidence of non-perioperative stroke?}

\section{METHODS}

L

Design: randomised controlled trial (MRC Asymptomatic Carotid Surgery Trial [ACST])

Allocation: concealed.*

Blinding: blinded (endpoint review committee).*

Follow up period: up to 5 years (mean 3.4 y).

Setting: 126 hospitals in 30 countries

Patients: 3120 patients (mean age 68 y, 66\% men) who had severe unilateral or bilateral carotid artery stenosis $(\geqslant 60 \%$ diameter reduction on ultrasonography) but no stroke or ischaemia in the previous 6 months, for whom both physician and patient were substantially uncertain whether to choose immediate CEA or deferral of any CEA until a more definite need for it was thought to have arisen. Exclusion criteria included known conditions that could preclude long term follow up, previous ipsilatera CEA, expectation of poor surgical risk (eg, because of acute myocardial infarction), and probable cardiac source of emboli.

$\mathbf{R}_{\mathbf{X}}$

Intervention: immediate CEA $(n=1560)$ or deferred CEA $(n=1560)$.

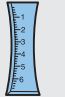

Outcomes: a composite outcome of perioperative mortality (caused by stroke or myocardial infarction) and morbidity (stroke), and incidence of non-perioperative stroke.

10 Patient follow up: all patients were included in the life table intention to treat analyses.

*See glossary.

\section{MAIN RESULTS}

About $90 \%$ and $10 \%$ of patients in the immediate and deferred CEA groups, respectively, received an ipsilateral CEA. At 5 years, the risk of the composite outcome as well as that of non-perioperative stroke was lower in the immediate CEA group than in the deferred CEA group (table). The overall risk per CEA of perioperative stroke or death was $3.1 \%$.

\section{CONCLUSION}

In patients with severe carotid artery stenosis but no recent ( $\leqslant 6 \mathrm{mo}$ ) stroke or ischaemia, immediate carotid endarterectomy (CEA) was

For correspondence: Dr A Halliday, ACST Office, St George's Hospital Medical School, London, UK. acst@sghms.ac.uk

Sources of funding: UK Medical Research Council and The Stroke Association. more effective than indefinite deferral of any CEA for reducing the net 5 year incidence of stroke.

\section{Abstract and commentary also appear in ACP Journal Club.}

\section{Commentary}

t is certainly clear that most patients with symptoms and $>70 \%$ carotid stenosis are better off with surgical than medical treatment. However, the data remain murky for asymptomatic patients, even with publication of the excellent ACST by Halliday et al. ${ }^{1}$

CEA is a nettlesome issue for internists, in part because the benefits of preventive "roto rooter" arterial cleaning seem obvious to many patients with carotid stenosis discovered in the course of a physical examination with subsequent testing. Before concluding that most asymptomatic patients with $\geqslant 60 \%$ diameter reduction on carotid ultrasonography should be referred to vascular surgeons, I would suggest that the following patient care protocols merit serious and open minded attention.

Firstly, because it is critically important in decision making to know if the carotid lesion is truly asymptomatic, I would suggest that such patients be referred in the first instance to an experienced neurologist, rather than a surgeon. Secondly, because the experience of technologists performing ultrasonography varies widely, computed tomographic angiography or magnetic resonance angiography should confirm the stenosis before surgery is considered. Thirdly, patients must recognize that they face only a $2 \%$ annual stroke rate with medical care, and that surgical morbidity and mortality is $3 \%$ among the most experienced surgeons. Fourthly, the surgical experience and track record of the surgeon to whom a patient is referred should be readily available, because operative complications may exceed the low rates of optimum care in research centres by $1 \%$ to $2 \%$.

Finally, some evidence (albeit anecdotal) suggests that the process of insidious carotid stenosis can be arrested and perhaps reversed by scrupulous control of risk factors. The jury is still out, but progression of carotid stenosis over time may be preventable, provided that the patient is strongly motivated and the doctor has excellent communication skills. ${ }^{2}$ Matthew Menken, MD Robert Wood Johnson Medical School New Brunswick, New Jersey, USA

1 Barnett HJ. Carotid endarterectomy. Lancet 2004;363:1486-7.

2 Furberg CD, Adams HP Jr, Applegate WB, et al. Effect of lovastatin on early carotid atherosclerosis and cardiovascular events. Circulation 1994;90:1679-87.

Immediate carotid endarterectomy (CEA) $v$ indefinite deferral of any CEA in severe carotid artery stenosis at 5 years*

\begin{tabular}{|c|c|c|c|}
\hline \multirow[b]{2}{*}{ Outcomes } & \multicolumn{2}{|c|}{ Kaplan-Meier risk estimates } & \multirow[b]{2}{*}{$\operatorname{RRR}(95 \% \mathrm{Cl})$} \\
\hline & Immediate CEA & Deferred CEA & \\
\hline Composite outcome & $6.4 \%$ & $11.8 \%$ & $46 \%$ (31 to 57 ) \\
\hline $\begin{array}{l}\text { Non-perioperative } \\
\text { stroke }\end{array}$ & $3.8 \%$ & $10.9 \%$ & $65 \%(54$ to 74$)$ \\
\hline
\end{tabular}

*Composite outcome = perioperative mortality (caused by stroke and myocardial infarction) and morbidity (stroke). Other abbreviations defined in glossary; RRR and $\mathrm{Cl}$ calculated from data in article. 